\title{
Perinatal long chain polyunsaturated fatty acid supply Are there long term consequences?
}

\author{
Hans DEMMELMAIR ${ }^{1}$ \\ Elvira LARQUE ${ }^{2}$ \\ Berthold KOLETZKO ${ }^{1}$ \\ ${ }^{1}$ Labor für Stoffwechsel und Ernährung, \\ Dr. von Hauner Children's Hospital, \\ Ludwig-Maximilians University of Munich, \\ Lindwurmstr. 4, D-80337 Muenchen, Germany \\ <Berthold.Koletzko@med.uni-muenchen.de> \\ ${ }^{2}$ Department of Animal Physiology, \\ Biological Faculty, University of Murcia, Spain
}

\begin{abstract}
Long-chain polyunsaturated fatty acids (LC-PUFA), especially docosahexaenoic acid (DHA), are essential components of biological membranes or act as precursors for eicosanoid formation, in case of the 20 carbon atom fatty acids, arachidonic acid (AA), dihomo- $\gamma$-linolenic acid and eicosapentaenoic acid. During pregnancy LC-PUFA are enriched in the fetal circulation relative to maternal plasma. The corresponding placental processes have not been fully elucidated so far, but there are good indications that the LC-PUFA enrichment during the materno-fetal transfer is mediated by differences in the incorporation into lipid classes within the placenta between fatty acids and that specific fatty acid binding and transfer proteins are of major importance. In vitro a plasma membrane fatty acid binding protein could be identified, which preferentially binds DHA and AA compared to linoleic and oleic acids; in addition the m-RNA expression of fatty acid transfer protein 4 (FATP-4) in placental tissue was found to correlate significantly with the DHA percentage in cord blood phospholipids. After birth the percentage of LC-PUFA in infantile blood rapidly declines to levels depending on the dietary LC-PUFA supply, although preterm and full-term babies can convert linoleic and $\alpha$-linolenic acids into $A A$ and $D H A$, respectively. Breast milk provides preformed LC-PUFA, and breastfed infants have higher LC-PUFA levels in plasma and tissue than infants fed formulas without LC-PUFA. The high percentage of DHA in brain and other nervous tissue and the fact that the perinatal period is a period of fast brain growth suggests the importance of placental DHA transfer and dietary DHA content for optimal infantile development. Most but not all randomized, double blind, controlled clinical trials in preterm and in healthy full term infants demonstrated benefits of formulas supplemented with DHA and AA for the neurological development compared to formulas without LC-PUFA. Furthermore, according to the concept of "metabolic programming" during the perinatal period a dietary factor, such as the availability of LC-PUFA, might have long term consequences. From the available data it has to be assumed that LC-PUFA are conditionally essential substrates during early life and a further investigation of the biological mechanisms related to their availability for the infant and of potential life long effects on cognitive function and potentially also risk factors for cardiovascular diseases and allergy are certainly warranted.
\end{abstract}

Key words: fatty acid transport protein, foetus, placenta, stable isotopes, infantile neurological development

ponent of structural phospholipids and serves as precursor for eicosanoids, which play important roles in cell division, signal transduction and many other physiological processes [3]. In preterm babies the availability of AA has been associated with weight at birth and growth during the first year of life [4]. Here we discuss some ideas about the mechanistic principles of the LC-PUFA transfer from the mother to the foetus via the placenta during pregnancy and its effect on the development of neural functions is addressed briefly.

\section{LC-PUFA supply in the perinatal period}

LC-PUFA of the $n-6$ and $n-3$ series are derived from the essential fatty acid precursors linoleic acid ( $n-6)$ and $\alpha$-linolenic acid ( $n-3)$ by con- secutive enzymatic desaturation and chain elongation [5]. LC-PUFA are incorporated into practically all tissues of the foetus and infant, and they are the predominant PUFA in mammalian brain and neuronal tissues [2]. In humans, most brain LC-PUFA are accumulated during the phase of rapid brain growth in the last trimester of gestation and the first 2 years after birth. Although the conversion of LC-PUFA from the essential fatty acid has been demonstrated in neonates [6], as other lipids they have to be transferred across the placenta to meet fetal demands [3]. The foetus needs to receive appreciable amounts of preformed $A A$ and DHA by placental transfer to meet LC-PUFA accretion rates in membrane rich tissues.

The accumulation of brain DHA and AA from dietary preformed $\mathrm{DHA}$ and $\mathrm{AA}$ is far more 
efficient than the accumulation from dietary essential fatty acid precursors and their endogenous desaturation and elongation. It is estimated that about half of postnatal brain AA accretion is derived from dietary preformed $A A$ in term baboon neonates [7], while fetal plasma DHA is about 8-20 fold more effective as a substrate for brain DHA accretion in fetal baboons compared with $\alpha$-linolenic acid [8]. Fetal LC-PUFA status depends on maternal intake, which has been demonstrated by an increase of cord blood DHA after maternal DHA supplementation during pregnancy $[9,10]$. On the other hand also the efficiency of the transfer seems to be an important factor, as differences in polyunsaturated fatty acid fetal-maternal relationships between appropriate for gestational age and intrauterine growth restricted foetuses have been observed [11].

\section{Placental transfer of fatty acids}

Numerous studies reported significant differences between the fatty acid composition of maternal plasma lipids at the time of delivery and cord plasma lipids. Percentages of saturated and monounsaturated fatty acids are very similar between maternal plasma phospholipids and cord blood phospholipids. On the other hand essential fatty acid percentages are significantly lower in cord blood phospholipids, which is accompanied by clearly increased LC-PUFA percentages [12]. These observations point to a preferential transfer of LC-PUFA by the placenta. This agrees with an in vitro observation after the incubation of immortalized trophoplast cells (BEWO) with radio labelled fatty acids [13]. After incubating BEWO cells in $200 \mu \mathrm{M}$ solutions of oleic acid, linoleic acid, AA or $\mathrm{DHA}$, respectively, at $37^{\circ} \mathrm{C}$ applying a fatty acid albumin ratio of 1:1 they found the highest cellular uptake for DHA and the lowest for oleic acid, while linoleic acid and AA were similar intermediate between the other studied fatty acids.

Using stable isotope labelled fatty acids we investigated the placental transfer in vivo in humans [14]. Palmitic-, oleic-, linoleic- and docosahexaenoic acids labelled with the natural carbon variant ${ }^{13} \mathrm{C}$ were administered orally 4 hours before elective caesarean section to pregnant women. Tracer concentration was analysed hourly in maternal blood samples until delivery, in cord blood and in placental tissue. The time course of tracer concentration in maternal samples can be considered an indicator of the availability of the tracer for placental transfer, thus the amount potentially available for transfer. On the other hand, the tracer concentration in placental tissue and cord blood, respectively, indicate the amount,

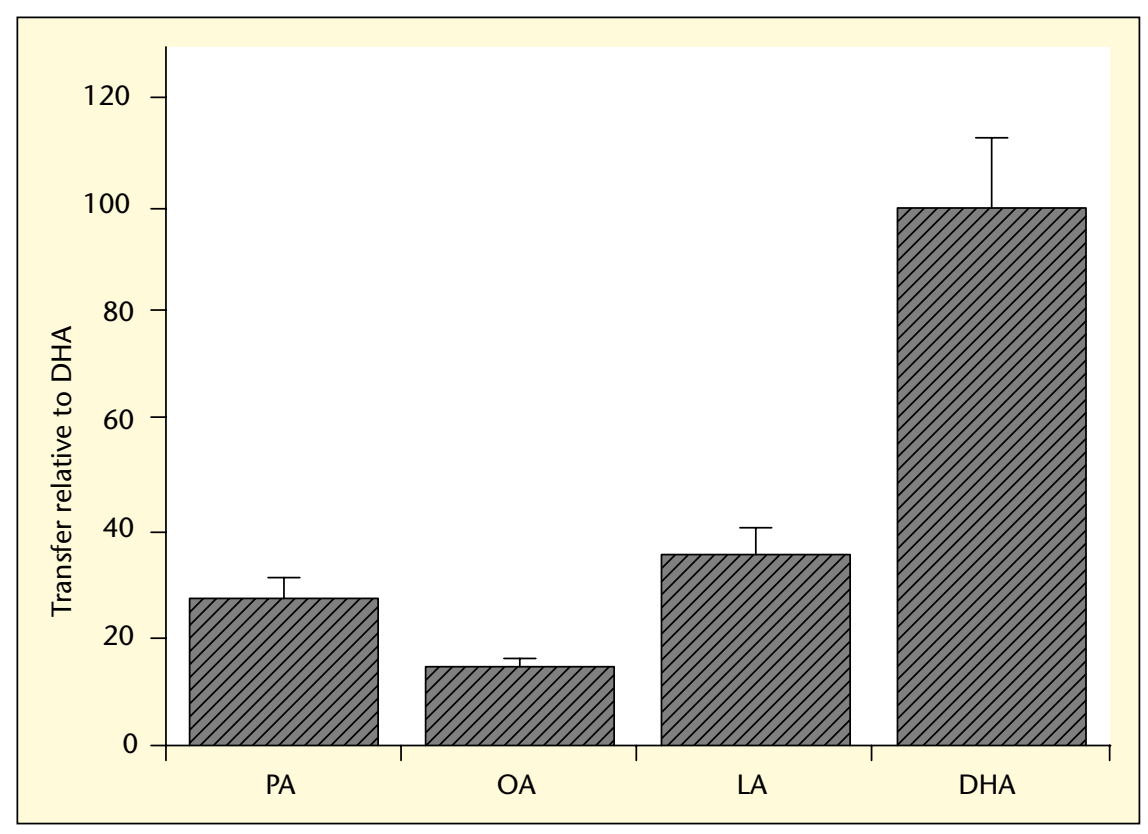

Figure 1. Comparison of the intensity of incorporation of palmitic acid (PA), oleic acid (OA), linoleic acid (LA) and docosahexaenoic acid (DHA) from maternal circulation into placental tissue four hours after oral intake of the fatty acids. Results ( $M \pm S E M, n=4)$ are expressed as percentage of DHA incorporation.

which has been transferred out of the maternal compartment into placenta or foetus, respectively. This has been related to the tracer concentration in the mother and the quotient obtained can be considered indicative for the transfer intensity of individual fatty acids. These quotients were not different between fatty acids, if cord blood tracer was related to the maternal compartment, but corresponding quotients for the ratio between placental tissue and maternal circulation were $7.1 \pm 1.0 \%$ $(\mathrm{M} \pm \mathrm{SEM}, \mathrm{n}=4)$ for palmitic acid, $3.8 \pm 0.4 \%$ for oleic acid, $9.2 \pm 1.3 \%$ for linoleic acid and $25.9 \pm 3.4 \%$ for $\mathrm{DHA}$, which was significantly higher than for the other fatty acids [14]. Although these percentages do not correspond to the real amount transferred, as this would require the inclusion of compartmental size into the calculation, a comparison between the studied fatty acids is valid, as compartmental size is the same for each of them. Thus it can be deduced that incorporation of DHA into placental tissue is 3 to 7 times more intensive than for the other studies fatty acids (figure 1). Although this can only be concluded for the time point studied (4 hours after intake) it seems to be at least qualitatively representative for the full process and the data agree with the in vitro observation.

The placental transfer of fatty acids is considered a complex process, which involves their binding to membrane proteins and cytoplasmatic transport proteins [15]. It is known that placenta takes up circulating non esterified fatty acids (NEFA) as well as NEFA released from maternal circulating lipids by placental lipases [16]. In addition, human placental tissue expresses receptors to VLDL, LDL and HDL lipoproteins [17], which could provide fatty acids to the placenta for transfer once they are released by intracellular lipases although the significance of this mechanism is still unknown.

NFA enter placental cells through both passive diffusion as well as via carrier proteins. This transport mechanism seems to involve plasma membrane fatty-acid binding protein (FABPpm/GOT2), fatty acid translocase (FAT/CD36), fatty acid transport proteins (FATP), and fatty acid binding proteins (FABP) [18]. Although the roles of these proteins in placental fatty acid uptake and further processing are not yet fully understood, it has been suggested that the enrichment of LC-PUFA in the fetal circulation relative to the maternal circulation is related to these proteins. A further fatty acid binding protein located exclusively on the maternal-facing membranes named placental plasma membrane fatty acid-binding protein $\left(p-F A B P_{p m}\right)$ has been proposed to be involved in the preferential uptake of LC-PUFA by trophoplast cells [19]. After the application of antibodies against placental plasma membrane fatty acid binding protein the uptake of DHA and AA was significantly more reduced than the uptake of oleic acid in BEWO cells, which points towards the importance of this postulated protein for a preferential uptake of LC-PUFA by the placenta [13]. However, definitive evidence about the structure and function 
of p-FABPpm must await analysis of its complete amino acid and/or CDNA sequence.

In the cytosol, fatty acids are esterified within the lipid fractions of the placenta or cross the tissue in either direction bound to cytosolic proteins. Also at this stage differences between DHA and other fatty acids become obvious, as only a small portion of DHA is found in the nonesterified fatty acids and in comparison to AA incorporation of DHA into triacylglycerols is high [13].

Several FABP are involved in the fatty acid transfer to the fetal circulation and so far heartFABP (H-FABP) and liver-FABP (L-FABP) and adipose tissue (A-FABP) have been identified in placenta. However, the mRNA expression of other cytosolic FABPs reported in other tissues might occur in placental tissue, which should be confirmed in trophoblast cells since placenta samples contain many cell types including a large content of blood cells.

In a double blind randomized intervention trial healthy pregnant women received during the second half of gestation supplements with modified fish oil providing $500 \mathrm{mg}$ DHA and $150 \mathrm{mg}$ of eicosapentaenoic acid per day with vitamins, or a placebo oil with vitamins. This led to a significantly higher percentage of DHA in the cord blood phospholipids of supplemented women $(9.6 \pm 2.2 \% \mathrm{wt} / \mathrm{wt}$ vs. $8.4 \pm 2.1 \% \mathrm{wt} / \mathrm{wt}$ ) [9]. In a group of 136 study participants the mRNA expression of various membrane bound and cytosolic fatty acid carrier proteins, $F A T / C D 36$, pm-FABP, A-FABP, B-FABP, H-FABP, FATP-1, FATP-6 and FATP-4 was investigated [20]. DHA supplementation did not induce a significant difference between groups in any of the fatty acid carriers, as might be expected from the known influence of DHA on PPAR expression and the known relationship between PPAR and some fatty acid carriers. Nevertheless, among the whole studied population there were significant positive correlations between the m-RNA expression for FATP-1 and placenta phospholipid DHA percentage $(r=0.36, p<0.001)$ and FATP-4 m-RNA expression and placental phospholipid $\mathrm{DHA}(r=0.39, p<0.001)$. DHA percentages in placental triacylglycerols correlated to FATP-1 and FATP-4 expression with r-values of 0.37 and 0.47 , respectively. DHA in triacylglycerols was also correlated to placental FAT and FABPpm although this association was less pronounced [20]. No corresponding significant correlations were observed for AA. Most important for placental transfer to the foetus seems the observation that the DHA percentage in cord blood phospholipids showed a weak, but significant correlation with the m-RNA expression for FATP-4 in the placenta [18]. Although these correlations might not fully explain the preferential transfer of LC-PUFA across the pla- centa, they clearly demonstrate the importance of the carrier proteins.

\section{LC-PUFA supply and outcomes}

Some, but not all studies have shown that maternal intake of fish, fish oils and n-3 LC-PUFA result in a slightly longer duration of gestation and a somewhat higher birth weight [10]. Recently meta-analyses of randomized controlled intervention trials were published reviewing effects of $n-3$ LC-PUFA supplementation of women with low risk and high risk pregnancies, respectively, on pregnancy outcomes $[21,22]$ The results show that $n-3$ LC-PUFA supplementation during pregnancy in general only to a small extent enhances pregnancy duration, but the risk of early preterm delivery is significantly reduced in high risk pregnancies (relative risk $0.39,95 \% \mathrm{Cl}$ $0.18,0.84$ ).

While a large number of studies have evaluated the influence of $n-3$ LC-PUFA supplementation on gestational length and birth weight, only few randomized studies have examined the effects of perinatal dietary LC-PUFA on neurodevelopment, but very recently in a large cohort study it could be shown that, low maternal seafood (the predominant source of n-3 LC-PUFA) intake during pregnancy was associated with increased risk of their children at age 8 years to be in the lowest quartile for the verbal intelligence quotient, suboptimum outcomes for prosocial behaviour, fine motor, communication, and social development scores [23].

Helland et al. performed a double blind randomized study with pregnant women receiving daily from 17-19 weeks of gestation until 3 months after delivery $1 \mathrm{~g}$ DHA provided with 10 $\mathrm{ml}$ cod liver oil, or a placebo oil (corn oil). No differences were reported on EEG scores at 3 months of age or Fagan test (as indicator of infant cognitive function) at 6 and 9 months of age in the babies [24]. In contrast, at the age of 4 years, children who were born to mothers who had received n-3 LC-PUFA during pregnancy and lactation had significantly higher IQ, as assessed with the Kaufman Assessment Battery for children (K-ABC) intelligence test [25]. Infants whose mothers had higher levels of DHA in red blood cells at birth showed more mature developmental profiles on singleobject attention measures and more optimal performance on distractibility assessments during the 2 years of life [26].

The current results show associations between early DHA status and cognitive function in infancy and early childhood. The participants of an expert workshop on dietary fat intake during the perinatal period held with support of the European Commission recommended that pregnant and lactating women should aim to achieving a dietary intake of n-3 LC-PUFA that supplies a DHA intake of at least $200 \mathrm{mg}$ per day. Intakes up to $1 \mathrm{~g}$ /day of DHA or 2.7 $\mathrm{g} /$ day of $\mathrm{n}-3$ LC-PUFA have been used in randomized trials without occurrence of significant adverse effects [27]. Women of childbearing age can meet the recommended intake of DHA by consuming one to two portions of sea fish per week, including fatty fish which is a good source of n-3 LC-PUFA. This intake of fatty fish rarely exceeds the tolerable intake of environmental contaminants. Furthermore, the worhshop participants found no evidence that women of childbearing age whose dietary intake of linoleic acid is adequate need an additional dietary intake of AA.

\section{Conclusions}

Fish oil supplementation during pregnancy slightly increases infant size at birth and significantly reduces early preterm birth before 34 weeks of gestation. Several studies have provided evidence for a link between early DHA status in the mother and cognitive development of her child. Given the great importance of these findings, further studies need to be performed to fully appreciate the effects and $\mathrm{n}-3$ fatty acid interventions in pregnancy, Such studies should also aim at a further elucidation of the active and preferential materno-fetal transfer of DHA across the placenta, apparently mediated by specific binding and transfer proteins.

Acknowledgements. The work reported herein has been carried out with partial financial support from the Commission of the European Communities, specific RTD Programme "Quality of Life and Management of Living Resources", within the $5^{\text {th }}$. Framework Programme, research grants no. QLRT-2001-00389 and QLK1-CT2002-30582, and the $6^{\text {th }}$. Framework Programme, contract no. 007036 "The early nutrition programming project", (www.metabolicprogramming.org). This manuscript does not necessarily reflect the views of the Commission and in no way anticipates the future policy in this area. BK is the recipient of a Freedom to Discover Award of the Bristol Myers Squibb Foundation, New York, NY, USA.

\section{REFERENCES}

1. FLEITH M, CLANDININ MT. Dietary PUFA for preterm and term infants: Review of clinical studies. Crit Rev Food Sci Nutr 2005; 45: 205-29.

2. INNIS SM. Dietary (n-3) fatty acids and brain development. / Nutr 2007; 137: 855-9.

3. LARQUE E, DEMMELMAIR H, KOLETZKO B. Perinatal supply and metabolism of long-chain polyunsaturated fatty acids: importance for the 
early development of the nervous system. In: Klimes I, Sebökova E, Howard BV, Ravussin E, eds. Lipids and insulin resistence. The role of fatty acid metabolism and fuel partitioning. 2002: 299-310.

4. KOLETZKO B, BRAUN M. Arachidonic acid and early human growth: Is there a relation? Ann Nutr Metab 1991; 35: 128-31.

5. SPRECHER H. Metabolism of highly unsaturated n-3 and n-6 fatty acids. Biochim Biophys Acta 2000; 1486: 219-31.

6. DEMMELMAIR H. V.SCHENCKU, BEHRENDTE, SAUERWALD T, KOLETZKO B. Estimation of arachidonic acid synthesis in full term neonates using natural variation of ${ }^{13} \mathrm{C}$ content. / Pediat Gastroenterol Nutr 1995; 21: 31-6.

7. WIJENDRAN V, HUANG MC, DIAU GY, BOEHM G, NATHANIELSZ PW, BRENNA JT. Efficacy of dietary arachidonic acid provided as triglyceride or phospholipid as substrates for brain arachidonic acid accretion in baboon neonates. Pediatr Res 2002; 51: 265-72.

8. SU HM, HUANG MC, SAAD NM, NATHANIELSZ PW, BRENNA JT. Fetal baboons convert 18:3n-3 to $22: 6 n-3$ in vivo. A stable isotope tracer study. I Lipid Res 2001; 42: 581-6.

9. KRAUSS-ETSCHMANN S, SHADID R, CAMPOY C, et al. Effects of fish-oil and folate supplementation of pregnant women on maternal and fetal plasma concentrations of docosahexaenoic acid and eicosapentaenoic acid: a European randomized multicenter trial. Am J Clin Nutr 2007; 85: 1392-400.

10. DECSIT, KOLETZKO B. N-3 fatty acids and pregnancy outcomes. Curr Opin Clin Nutr Metab Care 2005; 8: 161-6.

11. CETINI, GIOVANNININ, ALVINO G, et al. Intrauterine growth restriction is associated with changes in polyunsaturated fatty acid fetal-maternal relationships. Pediatr Res 2002; 52: 750-5.
12. BERGHAUS TM, DEMMELMAIR H, KOLETZKO B. Fatty acid composition of plasma lipid classes in maternal and cord plasma: conclusions for placental transfer. Eur J Pediatr 1998; 157: 31-6.

13. CAMPBELL FM, CLOHESSY AM, GORDON M], PAGE KR, DUTTA-ROY AK. Uptake of long chain fatty acids by human placental choriocarcinoma (BeWo) cells: role of plasma membrane fatty acid-binding protein. / Lipid Res 1997; 38: 2558-68.

14. LARQUE E, DEMMELMAIR H, BERGER B, HASBARGEN U, KOLETZKO B. In vivo investigation of the placental transfer of (13)C-labeled fatty acids in humans. / Lipid Res 2003; 44: 49-55.

15. DUTTA-ROY AK. Transport mechanisms for long-chain polyunsaturated fatty acids in the human placenta. Am / Clin Nutr 2000; 71: 315S-322S.

16. GAUSTER M, HIDEN U, BLASCHITZ A, et al. Dysregulation of placental endothelial lipase and lipoprotein lipase in intrauterine growthrestricted pregnancies. / Clin Endocrinol Metab 2007; 92: 2256-63.

17. WADSACK C, TABANO S, MAIER A, et al. Intrauterine growth restriction is associated with alterations in placental lipoprotein receptors and maternal lipoprotein composition. Am J Physiol Endocrinol Metab 2007; 292: E476E484.

18. LARQUE E, DEMMELMAIR H, KLINGLER M, DE IONGE S, BONDY B, KOLETZKO B. Expression pattern of fatty acid transport protein-1 (FATP1), FATP-4 and heart-fatty acid binding protein (H-FABP) genes in human term placenta. Early Hum Dev 2006.

19. CAMPBELL FM, DUTTA-ROY AK. Asymmetric distribution of the plasma membrane fatty acid-binding protein (FABPpm) in the human placenta. Biochem Soc Trans 1996; 24: 249S.
20. LARQUE E, KRAUSS-ETSCHMANN S, CAMPOY C, et al. Docosahexaenoic acid supply in pregnancy affects placental expression of fatty acid transport proteins. Am / Clin Nutr 2006; 84: 853-61.

21. HORVATHA, KOLETZKO B, SZAJEWSKA H Effect of supplementation of women in highrisk pregnancies with long-chain polyunsaturated fatty acids on pregnancy outcomes and growth measures at birth: a meta-analysis of randomized controlled trials. Br / Nutr 2007; 98: 253-9.

22. SZAJEWSKA H, HORVATH A, KOLETZKO B. Effect of $n-3$ long-chain polyunsaturated fatty acid supplementation of women with low-risk pregnancies on pregnancy outcomes and growth measures at birth: a meta-analysis of randomized controlled trials. Am J Clin Nutr 2006; 83: 1337-44.

23. HIBBELN JR, DAVIS JM, STEER C, et al. Maternal seafood consumption in pregnancy and neurodevelopmental outcomes in childhood (ALSPAC study): an observational cohort study. Lancet 2007; 369: 578-85.

24. HELLAND IB, SAUGSTAD OD, SMITH L, et al. Similar effects on infants of $n-3$ and $n-6$ fatty acids supplementation to pregnant and lactating women. Pediatrics 2001; 108: E82.

25. HELLAND IB, SMITH L, SAAREM K, SAUGSTAD OD, DREVON CA. Maternal supplementation with very-long-chain $\mathrm{n}-3$ fatty acids during pregnancy and lactation augments children's IQ at 4 years of age. Pediatrics 2003; 111: e39-e44.

26. COLOMBO ], KANNASS KN, SHADDY D], et al. Maternal DHA and the development of attention in infancy and toddlerhood. Child Dev 2004; 75: 1254-67.

27. KOLETZKO B, CETIN I, BRENNA JT. Dietary fat intakes for pregnant and lactating women. $\mathrm{Br}$ ) Nutr 2007; (in press). 